\title{
Knowledge, attitudes and practices towards malaria diagnostics among healthcare providers and healthcare seekers in Kondoa district, Tanzania: a multi- methodological situation analysis
}

\section{Leah F. Bohle ( $\sim$ leah.bohle@swisstph.ch )}

Swiss Tropical and Public Health Institute

\section{Ally-Kebby Abdallah}

Health Promotion and System Strengthening Project (HPSS)

Francesco Galli

Veterinary Public Health Institute, University of Bern

\section{Robert Canavan}

Swiss Tropical and Public Health Institute

\section{Kate Molesworth}

Swiss Tropical and Public Health Institute

\section{Research Article}

Keywords: Malaria, Tanzania, Knowledge, Attitudes, Practices, Healthcare providers, Healthcare seekers, Malaria diagnostics, Rapid diagnostic test, mRDT

Posted Date: February 25th, 2022

DOI: https://doi.org/10.21203/rs.3.rs-1389226/v1

License: (c) (i) This work is licensed under a Creative Commons Attribution 4.0 International License. Read Full License 


\section{Abstract}

Background: Despite the large-scale rollout of malaria rapid diagnostic tests (mRDTs) in Tanzania, many healthcare providers (HCPs) continue using blood film microscopy (BFM) and clinical examination to diagnose malaria, which can increase the risk of mal-diagnosis and over prescribing of anti-malarials. Patients disregarding negative test results and self-treating exacerbate the problem. This study explored HCPs' and healthcare seekers' knowledge, attitudes and practices regarding mRDTs in comparison to BFM testing.

Methods: The study was located in Kondoa District, Dodoma Region, Tanzania. A multi-methodological approach was adopted including i) a health facility inventory and screening of logbooks from May 2013 to April 2014 with 77,126 patient entries from 33 health facilities; ii) a survey of 40 HCPs offering malaria services; and iii) a survey of 309 randomly selected household members from the facilities' catchment area. Surveys took place in April and May 2014.

Results: Health facility records revealed that out of 77,126 patient entries, $22 \%(n=17,235)$ obtained a malaria diagnosis. Of those, $45 \%$ were made with BFM, 33\% with mRDT and $22 \%$ with clinical diagnosis. A higher rate of positive diagnoses was observed with BMF compared with mRDT (71\% vs $14 \%$ ). In the HCP survey, $48 \%$ preferred using BMF for malaria testing, while $52 \%$ preferred mRDT. Faced with a negative mRDT result for a patient presenting with symptoms typical for malaria, $25 \%$ of HCPs stated they would confirm the result with a microscopy test, $70 \%$ would advise or perform a clinical diagnosis and $18 \%$ would prescribe antimalarials. Interviews with household members revealed a preference for microscopy testing (58\%) over mRDT (23\%), if presented with malaria symptoms. For participants familiar with both tests, a second opinion was desired in $45 \%$ after a negative microscopy result and in $90 \%$ after an mRDT.

Conclusions: Non-adherence to negative diagnostics by HCPs and patients continues to be a concern. Frequent training and supportive supervision for HCPs diagnosing and treating malaria and non-malaria febrile illnesses is essential to offer quality services that can instil confidence in HCPs and patients alike. The introduction of new diagnostic devices should be paired with context-specific behaviour change interventions targeting healthcare seekers and healthcare providers.

\section{Background}

The new millennium saw a significant scaling up of malaria control interventions in sub-Saharan Africa, initiated by numerous international donors and in line with the United Nations Millennium Development Goals, the Roll Back Malaria Partnership objectives and the World Health Organization's (WHO) Global Technical Strategy 2016-2030 [1-4]. Despite the success that these interventions have had on reducing, and in some cases eliminating, malaria infection related morbidity and mortality, in recent years progress has stalled calling for new, targeted and innovative measures to further the positive advancements made and to avoid regression $[2,5]$.

According to the 2020 World Malaria Report, the WHO African Region, accounted for 94\% of global malaria cases in 2019. The same year, six African countries (i.e. Nigeria, the Democratic Republic of the Congo, the United Republic of Tanzania (hereinafter Tanzania), Mozambique, Niger and Burkina Faso) accounted for half of all malaria deaths globally [2]. Tanzania has a population of almost 60 million [6]; approximately $93 \%$ of the population live in malaria transmissible areas and $96 \%$ of infections are due to Plasmodium falciparum [7]. The WHO estimated that there were over six million cases of malaria in Tanzania in 2019 and more than 20,000 malaria related deaths [2]. Since malaria is preventable and treatable, effective case management requires prompt access to diagnosis and

Page $2 / 22$ 
treatment, provider compliance to malaria treatment guidelines and patient adherence to medication [8-10]. This in turn can help to reduce the spread of antimalarial drug resistance, reduce unnecessary use of limited resources and better identify non-malaria febrile illnesses $[8,11]$. The WHO guidelines for the treatment of malaria recommend testing all patients with suspected malaria prior to medical treatment [8]. Historically, diagnosis and treatment of malaria in Tanzanian public health facilities was presumptive [12], often leading to over diagnosis of malaria and an incorrect diagnosis for patients suffering with symptoms similar to malaria both resulting in over-prescription of anti-malarials [10,13-15]. Fortunately, the rate of unconfirmed malaria diagnoses in Tanzania has gradually been declining, according to the Health Management Information System, from 36\% in 2014 to 2\% in 2018 [16].

As of 2020, diagnosis of malaria via microscopic examination in Tanzania's public sector is available in 20\% of all public health facilities including regional and district hospitals and health centres [16]. However, there is a real concern of malaria being over-diagnosed when using microscopy as a sole diagnostic test [17]. The quality of malaria microscopy is dependent upon, e.g., the availability and competence of a skilled laboratory microscopist, quality assurance systems in place and electricity to power the microscope [18]. Given the simplicity and cost effectiveness of malaria rapid diagnostic tests (mRDTs), together with their proven reliability, mRDTs have become the most practical and suitable tool for malaria diagnosis in Tanzania, especially in remote rural areas, where resources are limited [18-24].

Previous studies in Tanzania have revealed reasonable to high levels of knowledge about the seriousness of malaria, its symptoms and preventive measures among the population and a high knowledge of the availability of artemisinin-based combination therapy (ACT) [25-31]. In contrast, and since the large-scale rollout of mRDTs in Tanzania in 2009, very little research has been conducted to provide information about the knowledge, attitudes and practices towards malaria diagnostics among HCPs and healthcare seekers alike [32-36]. The few existing studies and reports have documented a concerning disregard for negative diagnostic test results, especially when using mRDTs, among healthcare seekers and some HCPs who continue to prescribe antimalarial medication despite negative test results $[15,36,37]$. Furthermore, two studies showed that trained HCPs appeared to be less compliant with mRDT results compared with lower cadres of healthcare providers. Over-confidence in their awareness of clinical symptoms and previous experience were considered reasons for non-compliance with mRDT results [10, 36, 38].

In 2011, The Health Promotion and System Strengthening (HPSS) project was launched in Tanzania. The project is implemented by the Swiss Tropical and Public Health Institute (Swiss TPH) and is funded by the Swiss Agency for Development and Cooperation (SDC). The project aims to support the Tanzanian Government to strengthen the health system countrywide and thereby advance towards Universal Health Care and Tanzania's Development Vision 2025. The objective of the HPSS project is to apply a comprehensive approach to health system strengthening within the domains of health promotion, health financing, technology management and medicines management [39-41]. This study was conducted as part of the health promotion focal area. The aim was to explore the impact that the roll-out of mRDTs had among healthcare providers and seekers in Kondoa district and to explore their knowledge, attitudes and practices regarding mRDTs in comparison to microscopic malaria diagnostic testing.

\section{Methods}

\section{Study setting}

The study, here reported, was located in Kondoa District, in the north of Dodoma Region in central Tanzania. In the year prior to the study, the population was approximately 270,000 and the district was divided into 28 wards (a town, 
partial town or collection of villages), including 96 villages and 12 hamlets (a sub-divided village) (41). Agriculture and livestock constituted the inhabitants' predominant source of income.

\section{Study overview}

A multi-methodological approach was adopted and included i) a health facility inventory and systematic screening of facility logbooks conducted over the 12-month period preceding the study; ii) a survey of healthcare providers who were offering malaria related services; and iii) a survey of household members.

\section{Sample, sampling process and methodology}

\section{Health facility inventory}

Data from health facility logbooks were analysed over a 12-month period, i.e., between April/May 2013 to April/May 2014. A one-year period was chosen to provide a sound database and reflection of all seasonal fluctuations throughout the year. Thirty-three facilities were included from the intended 41 public (i.e. dispensaries, health centres and a district hospital) and private health facilities (i.e. faith-based facilities, private laboratories) offering malaria counselling and diagnosis throughout Kondoa District. Some facilities had to be excluded owing to missing logbooks or entries, lack of readability or higher levels of confidentiality (e.g. military-run). The total number of patients per month, the number of mRDTs and blood films conducted per month, the test results, the diagnosis and treatment prescribed were counted and cross-checked by a second data collector. In addition, a retrospective and real-time stock count of available malaria tests was conducted in each of the facilities also dating back up to one year and, where available, the microscopes were tested for functionality. All counts were then entered into an SPSS database.

\section{Survey of healthcare providers at facility level}

All HCPs involved in counselling and testing for malaria at dispensary and health centre levels and two HCPs randomly selected from the District Hospital were asked to participate in the study and undergo an interview. A total of $40 \mathrm{HCPs}$ were included in the study. The survey questionnaire was paper-based, semi-structured and translated into Swahili. The questionnaire included closed and few qualitative open-ended questions, which included sociodemographic questions and questions related to knowledge, attitudes and practices related to malaria diagnosis.

\section{Survey of healthcare seekers at household level}

A two-stage cluster survey approach was used for the village sampling and household survey. Household members were eligible for inclusion in the study if they i) were over the age of 16 years; ii) had undergone a malaria test within 12 months at a health facility in Kondoa District at the time of data collection; iii) were in a stable health condition; and iv) had provided consent. The villages were randomly selected from the catchment area of the selected health facilities. The survey team began at the centre of the village, which was divided into four main clusters. A bottle and coin method (equal to the bottle or pen method) was used to randomly select households [42]. The first household was visited; thereafter every forth household opposite the previous household was visited. This procedure was repeated until either a junction or the border of the village was reached. The bottle and coin technique was then reemployed and the process resumed until the required number of households per village had been sampled. The 309 household members who agreed to participate were asked to read an information sheet in Swahili and sign a consent form. Where a participant was unable to read or sign their name, an impartial witness accompanying the process, informed participants and then invited participants to provide fingerprint consent. As for 
the HCPs' survey, the household survey questionnaire was paper-based, semi-structured including closed and openended questions and translated to Swahili.

\section{Data collection}

A team of five Tanzanian data collectors (three female and two male) were selected. They were fully informed on the scope of the study, the ethics and use of information and consent forms. In addition, they were trained on interviewing and completing survey questionnaires, recording data, quality issue concerns and data entry. A pilot study was conducted in December 2013 to validate the survey questionnaire. Reponses to the qualitative questions were coded manually using in-vivo codes and provided as closed reply options in the final questionnaire. Final data collection took place between 6 January and 8 February 2014.

\section{Data analysis}

Quantitative data analysis was performed with Stata, version 12, and descriptive analyses were conducted on all variables. For categorical variables, the results were reported in frequency tables with sample sizes and proportions. If a survey question allowed multiple answers, those were ranked by frequency. Continuous variables were summarized by using their mean, minimum and maximum.

Given the limited sizes of the different samples, simple exploratory methods were used to assess association between variables of interest. In the case of associations between two categorical variables, Pearson's Chi-square test was used if the expected number of observations in all sub-categories was higher than five, whereas Fisher's exact test was preferred if the number of expected observations in at least one sub-category was five or less. Correspondingly, p-values, here reported, were derived from Pearson's Chi-square or Fisher's test. If the association was significant, odds ratios (OR) were calculated and reported together with their $95 \%$ confidence interval (Cl). A pvalue for the odds ratio was calculated with either Pearson's Chi-square or Fisher's exact tests and was reported after the estimate.

When testing association between a continuous and a categorical variable, the unpaired t-test was used if the sample size of each sub-group was of at least 30 and if the observations were normally distributed (normality was assessed with the Skewness and Kurtosis test), whereas the Mann-Whitney rank sum test was used when any of the two assumptions were not true. Results were reported as group averages and the corresponding $95 \% \mathrm{Cl}$ and $\mathrm{p}$ values. In case of direct comparison of proportions between two different samples, the two-sample test of proportion was used and the p-value of the Z-statistic was reported. Qualitative open-ended questions from both final survey questionnaires were coded in line with the semi-structured questionnaire, using in-vivo codes and followed by thematic grouping.

\section{Results}

\section{Retrospective data collection in health facilities}

\section{Malaria diagnosis}

Thirty-two of the 33 study health facilities provided their logbooks. These included 25 dispensaries, five health centres, one laboratory and one district hospital, reporting a total of 77,126 patient entries over a 12-month period (Table 1). Among them, $22 \%$ obtained a malaria diagnosis, $45 \%$ of all those diagnosed were tested via blood film 
microscopy (BFM), 33\% with mRDT and $22 \%$ through clinical diagnosis alone. Most patients obtained a diagnosis from the primary health care level, i.e., a dispensary (39\%) or health centre (30\%). The proportion of patients obtaining a malaria diagnosis out of the total number of patients visiting a facility was highest in the laboratories (57\%), followed by health centres (31\%), dispensaries (20\%) and finally the district hospital (10\%). At the district hospital, all malaria diagnoses were based on mRDT. In contrast, mRDTs were used less frequently in dispensaries (37\%) and health centres (20\%), and not at all in laboratories.

The mRDTs were used most frequently in government-owned facilities ( $n=5044 ; 88 \%)$, followed by faith-based organisations ( $n=665 ; 12 \%$ ) and not used at all in the two privately owned facilities assessed. Blood film microscopy was the only test that was used in laboratories and represented $58 \%(n=2965)$ of all tests in health centres and 24\% ( $n=1609)$ in dispensaries. Microscopy use was not reported from the district hospital. Microscopy was used exclusively by private facilities and at $63 \%$ by faith-based organisation facilities, whereas governmentowned facilities reported $9 \%$ use. Regardless of the diagnostic method used, the proportion of malaria positive cases diagnosed among those who received a diagnosis was $58 \%$ (Table 1) with a notably low proportion at the district hospital (3\%).

The 24 government-owned health facilities conducted the highest number of malaria tests overall $(n=9217), 51 \%$ of which tested positive (Table 1), while the two private health facilities conducted 5176 malaria tests overall, with $77 \%$ testing positive. Faith-based organisations conducted the least number of malaria tests $(n=2842)$ and had the lowest proportion of positive diagnoses at $40 \%$. Overall, a much higher positive test rate was observed with microscopy testing (71\%) than with mRDT (14\%). This $57 \%$ difference (95\% Cl: $55.6-58.4)$ was found to be statistically significant $(p<0.001)$.

Table 1 Malaria diagnoses displayed in numbers and proportions, stratified by facility type, ownership and diagnostic procedure 


\begin{tabular}{|c|c|c|c|c|c|c|c|c|}
\hline & $\begin{array}{l}\text { Overall } \\
(\mathrm{N}=33)\end{array}$ & $\begin{array}{l}\text { Dispensary } \\
(\mathrm{n}=25)\end{array}$ & $\begin{array}{l}\text { Health } \\
\text { centre } \\
(n=5)\end{array}$ & $\begin{array}{l}\text { District } \\
\text { hospital } \\
(n=1)\end{array}$ & $\begin{array}{l}\text { Laboratory } \\
(\mathrm{n}=1)\end{array}$ & $\begin{array}{l}\text { Government } \\
(\mathrm{n}=24)\end{array}$ & $\begin{array}{l}\text { Faith- } \\
\text { based } \\
(n=6)\end{array}$ & $\begin{array}{l}\text { Private } \\
(n=2)\end{array}$ \\
\hline Total patients & 77126 & 33439 & 16587 & 21430 & 5670 & 59343 & 8737 & 9046 \\
\hline $\begin{array}{l}\text { Total malaria } \\
\text { diagnoses }\end{array}$ & $\begin{array}{l}17235 \\
(22.3)\end{array}$ & $\begin{array}{l}6661 \\
(19.9)\end{array}$ & $\begin{array}{l}5135 \\
(31.0)\end{array}$ & $\begin{array}{l}2218 \\
(10.3)\end{array}$ & $\begin{array}{l}3221 \\
(56.8)\end{array}$ & $9217(15.5)$ & $\begin{array}{l}2842 \\
(32.5)\end{array}$ & $\begin{array}{l}5176 \\
(57.2)\end{array}$ \\
\hline Positive cases & $\begin{array}{l}9904 \\
(57.5)\end{array}$ & $\begin{array}{l}3715 \\
(55.8)\end{array}$ & $\begin{array}{l}4021 \\
(78.3)\end{array}$ & $64(2.9)$ & $\begin{array}{l}2104 \\
(65.3)\end{array}$ & $4781(51.9)$ & $\begin{array}{l}1122 \\
(39.5)\end{array}$ & $\begin{array}{l}4001 \\
(77.3)\end{array}$ \\
\hline $\begin{array}{l}\text { mRDT } \\
\text { diagnoses }\end{array}$ & $\begin{array}{l}5709 \\
(33.1)\end{array}$ & $\begin{array}{l}2473 \\
(37.1)\end{array}$ & $\begin{array}{l}1018 \\
(19.8)\end{array}$ & $\begin{array}{l}2218 \\
(100.0)\end{array}$ & $0(0.0)$ & $5044(54.7)$ & $\begin{array}{l}665 \\
(23.4)\end{array}$ & $0(0.0)$ \\
\hline Positive cases & $\begin{array}{l}786 \\
(13.8)\end{array}$ & $500(20.2)$ & $\begin{array}{l}222 \\
(21.8)\end{array}$ & $64(2.9)$ & - & $709(4.1)$ & $\begin{array}{l}77 \\
(11.6)\end{array}$ & - \\
\hline Negative cases & $\begin{array}{l}4877 \\
(85.4)\end{array}$ & $\begin{array}{l}1940 \\
(78.4)\end{array}$ & $\begin{array}{l}783 \\
(76.9)\end{array}$ & $\begin{array}{l}2154 \\
(97.1)\end{array}$ & - & $4300(85.2)$ & $\begin{array}{l}577 \\
(86.8)\end{array}$ & - \\
\hline Invalid tests & $\begin{array}{l}46 \\
(0.8)\end{array}$ & $33(1.3)$ & $\begin{array}{l}13 \\
(1.3)\end{array}$ & $0(0.0)$ & - & $35(0.7)$ & $\begin{array}{l}11 \\
(1.7)\end{array}$ & - \\
\hline $\begin{array}{l}\text { Non readable } \\
\text { tests }\end{array}$ & $0(0.0)$ & $0(0.0)$ & $0(0.0)$ & $0(0.0)$ & - & $0(0.0)$ & $\begin{array}{l}0 \\
(0.0)\end{array}$ & - \\
\hline $\begin{array}{l}\text { Blood } \\
\text { film/microscopy } \\
\text { diagnoses }\end{array}$ & $\begin{array}{l}7795 \\
(45.2)\end{array}$ & $\begin{array}{l}1609 \\
(24.2)\end{array}$ & $\begin{array}{l}2965 \\
(57.7)\end{array}$ & $0(0.0)$ & $\begin{array}{l}3221 \\
(100)\end{array}$ & $819(8.9)$ & $\begin{array}{l}1800 \\
(63.3)\end{array}$ & $\begin{array}{l}5176 \\
(100)\end{array}$ \\
\hline Positive cases & $\begin{array}{l}5518 \\
(70.8)\end{array}$ & $754(46.9)$ & $\begin{array}{l}2660 \\
(89.7)\end{array}$ & - & $\begin{array}{l}2104 \\
(65.3)\end{array}$ & $725(88.5)$ & $\begin{array}{l}792 \\
(44.0)\end{array}$ & $\begin{array}{l}4001 \\
(77.3)\end{array}$ \\
\hline Negative cases & $\begin{array}{l}2226 \\
(28.6)\end{array}$ & $821(51.0)$ & $\begin{array}{l}288 \\
(9.7)\end{array}$ & - & $\begin{array}{l}1117 \\
(34.7)\end{array}$ & $82(10.0)$ & $\begin{array}{l}969 \\
(53.8)\end{array}$ & $\begin{array}{l}1175 \\
(22.7)\end{array}$ \\
\hline Invalid tests & $\begin{array}{l}49 \\
(0.6)\end{array}$ & $34(2.1)$ & $\begin{array}{l}15 \\
(0.5)\end{array}$ & - & $0(0.0)$ & $12(1.5)$ & $\begin{array}{l}37 \\
(2.1)\end{array}$ & $0(0.0)$ \\
\hline $\begin{array}{l}\text { Non readable } \\
\text { tests }\end{array}$ & $2(0.1)$ & $0(0.0)$ & $2(0.1)$ & - & $0(0.0)$ & $0(0.0)$ & $\begin{array}{l}2 \\
(0.1)\end{array}$ & $0(0.0)$ \\
\hline $\begin{array}{l}\text { Clinical } \\
\text { diagnoses }\end{array}$ & $\begin{array}{l}3731 \\
(21.7)\end{array}$ & $\begin{array}{l}2579 \\
(38.7)\end{array}$ & $\begin{array}{l}1152 \\
(22.4)\end{array}$ & $0(0.0)$ & $0(0.0)$ & $3354(36.4)$ & $\begin{array}{l}377 \\
(13.3)\end{array}$ & $0(0.0)$ \\
\hline
\end{tabular}

\section{Malaria test availability}

All health facilities had at least one type of malaria test in stock, 35\% $(n=14)$ of them had both mRDTs and microscopes, whereas $58 \%(n=23)$ had only mRDTs and $8 \%(n=3)$ only microscopes. On average, mRDTs as diagnostic tools were available in $63 \%$ of the health facilities over a 12-month period, although their availability varied in time with an overall constant increase in the frequency of stock-outs between May 2013 and April 2014 (Fig. 1). MRDT availability in government-owned health facilities was, on average, $64 \%$ over the 12 -month period but only $44 \%$ during the rainy season (December to April) when malaria incidence increases. The majority of healthcare workers reported mRDT stock-outs $(n=33 ; 83 \%)$ with delays in delivery being the most frequently stated reason. 
MRDT availability significantly varied between dispensaries (70\%) and health centres (42\%), i.e., mRDTs were 3.2 times more likely to be available in dispensaries than in health centres (95\% $\mathrm{Cl}: 1.6-6.3 ; \mathrm{p}<0.001)$. The hospital and laboratory were excluded from categorization because there were too few observations ( 9 and 12, respectively). Comparisons between government versus faith-based organisation ownership did not show any significant differences regarding mRDT availability. Comparison with private facilities was not feasible owing to a lack of relevant information (e.g. seven observations from one health facility).

Information regarding BFM testing availability was incomplete because the physical inventory for BFM was only performed in 116 of the 309 data months. However, based on the available data, blood films and microscopes were available in $91 \%$ of health facilities and their availability was constant over time. Any further comparisons of microscope availability between categories of health facility level and ownership was not feasible owing to a lack of data.

\section{Survey with healthcare providers}

\section{Socio-demographic background of interviewees and setting}

The majority of HCPs interviewed were females (63\%) (Table 2). A total of 35\% were nurses, followed by medical attendants (28\%) and their mean work experience was 19.2 years (min. 7 months, max. 41 years). The great majority of HCPs were working in a dispensary (83\%). HCPs interviewed declared that they performed laboratory diagnosis of malaria (93\%), consulted patients (75\%) and assisted in consultations (53\%).

Table 2 Socio-demographic setting and activity profile of healthcare providers interviewed

\section{Malaria perception in the area of service}

Of the participating HCPs, $73 \%$ claimed to perceive malaria as a minor (or average) health concern, while only $28 \%$ answered that they considered malaria a major health concern. Among the latter, the two most cited reasons were the "high number of patients" ( $n=10)$ and the "low knowledge of prevention" $(n=8)$. Among the 29 HCPs perceiving malaria as a minor concern, the most frequent reasons given were "high knowledge of prevention" $(n=26)$, "high knowledge of malaria" ( $n=19)$, "low number of patients" $(n=18)$, “low risk of infection" ( $n=16)$, "no common disease any longer" $(n=16)$ and "many negative results" $(n=11)$.

\section{Training for malaria diagnostic}

More than three quarters of respondents reported that they had received at least one type of training for the use of mRDTs $(n=31 ; 78 \%)$. Twenty were trained by the district medical officer at the district level, 10 by laboratory technicians, 6 by co-workers, 3 at college, 1 by a clinical officer and 1 was trained in "another" way. In contrast, 20\% $(n=8)$ did not receive any training and one person could not remember if they had received any such training. Out of

the $30 \mathrm{HCPs}$ who responded to a question concerning the number of times they received mRDT training, the majority stated that they received training once $(n=25 ; 83 \%)$, two were trained twice and three received training on three or more occasions. The last time any of the 31 respondents received mRDT training was on average 3.4 years before data collection commenced in 2015 (min. 2 years, max. 5 years). Finally, almost all respondents declared that they felt the need for further mRDT training $(n=37 ; 93 \%)$. The most frequently cited reasons for needing further mRDT training were "to improve the quality of the service" ( $n=33)$, "to improve knowledge" $(n=27)$, "to improve the 
Observations $(\mathbf{N}=\mathbf{4 0}) \quad \% \quad$ accuracy of the test result" $(n=24)$ and "to Age (years)

Younger than 30

30-39

40-49

50 or older

\section{Sex}

\section{Female \\ Male \\ Profession}

Nurse

14

Medical Attendant

Clinical Officer

Laboratory Assistant

Medical Doctor

\section{Work experience (years)}

Less than five

10

5-19

20-29

30 or more

\section{Level of health facility}

Dispensary 33

Health Center

District Hospital

Private Laboratory

\section{Ownership of health facility}

Government

28

Faith-based

Private

$$
8
$$

4

\section{Location of health facility}

Village
Ward headquarters

20.0 for malaria diagnosis. At the time of data

15.0 collection, the last time respondents were 22.5 trained to use BFM for malaria diagnosis was on average 12.7 years earlier.

42.5

Knowledge, attitudes and perceptions towards malaria diagnosis

62.5

37.5

35.0

Inquiring on the preference of malaria testing

method, almost half of HCPs ( $n=19 ; 48 \%$ )

claimed to prefer using microscopy for testing for malaria while $43 \%$ ( $n=17)$ preferred using mRDT. Among the former, the three most

27.5 frequently given reasons for their preference

17.5

12.5 were "trust in blood slide" ( $n=19 ; 100 \%)$, "old/well known test" ( $n=12 ; 63 \%)$, the microscopic test being "more accurate" ( $n=11$;

$7.558 \%)$ and "no trust in mRDT" ( $n=8 ; 42 \%)$. The most frequent reasons given for a stated preference for mRDT were that they were 25.0 "simple to use" ( $n=16 ; 94 \%)$, they provide a 20.0 "quick test and result" ( $n=15 ; 88 \%)$, that they "trust in mRDT" ( $n=13 ; 77 \%)$ and that they are "more accurate" compared to microscopic 25.0 30.0 diagnosis $(n=6 ; 35 \%)$. Specifically inquiring on trust, the majority of all HCPs ( $n=35,88 \%)$ declared that they trusted $\mathrm{mRDT}$ results
82.5

10.0

5.0

2.5

70.0

20.0

10.0

55.0

25.0 because i) they trusted the accuracy of the test (94\%); ii) the accuracy was proven by the government (76\%); iii) it is easy to use (67\%); and iv) using mRDTs was an "instruction by the government" 40\% $(n=13)$. Five respondents $(13 \%)$ stated that they did not trust the accuracy of the test result because of an issue with false negative results.

When considering only the HCPs that answered the question on test preference (36 out of 40), test preference seemed to vary significantly with the profession of the respondent $(p=$ 0.046). Nurses, clinical officers and medical doctors tended to prefer mRDT to microscopy 
District town

\section{Malaria-related tasks at health facility}

\begin{tabular}{ll}
\hline Laboratory diagnosis of malaria & 37 \\
\hline Consultation of patients & 30 \\
\hline Other & 25
\end{tabular}

Assistance in consultation
8 37 30 25
20.0 testing (64\%, $67 \%$ and $71 \%)$, whilst the majority of laboratory assistants and medical

92.5 attendants preferred microscopy testing over mRDT (60\% and $90 \%$, respectively). The stated 75.0 preference also varied significantly by type of 62.5 52.5 malaria tests available at the health facility $(p=$ 0.014). Among those facilities where both tests were available, $77 \%$ of health workers stated a preference for mRDT, whereas among those

facilities where only mRDT or only microscopy were available, $30 \%$ stated a preference towards mRDT. If only one of the two diagnostic tests were available in the health facility, health workers were $87 \%$ less likely to prefer mRDT compared with health workers of health facilities where both tests were available (OR: 0.13 [95\% $\mathrm{Cl} 0.019-0.761]$, $p$ $=0.009)$. Among those who declared that they had never received mRDT training $(n=8), 71 \%$ favoured mRDT and $29 \%$ favoured microscopy. In contrast, among those who received mRDT training $(n=28)$ the preference was less clear: $57 \%$ claimed to prefer microscopy and $43 \%$ claimed to prefer mRDT.

Inquiring on the practice of confirming diagnostic test results: in the case of a positive mRDT result, $48 \%$ of respondents reported that they would advise confirmation with microscopy, whereas 53\% would not advise it (Table 3 ). The top reason for advising confirmation with blood films were "trust in blood slide" (95\%), while the main reasons given for not advising confirmation with microscopy were "trust in result" (95\%) and "trust in mRDT" (86\%). In the case of a negative mRDT result, the majority of the respondents (68\%) would advise confirmation with microscopy. The top reason for advising such confirmation was "trust in microscopy" (92\%), while the main reasons for not advising so, were "trust in result" (100\%), and "trust in mRDT" (92\%). HCPs who preferred mRDT were six times more likely against advising confirmation of a negative mRDT result with microscopy when compared with HCPs that preferred microscopy testing. Furthermore, all those who did not trust the accuracy of the mRDT result (n = 5) advised confirmation of a negative mRDT with microscopy. In the explicit case of a negative mRDT result for a patient presenting with malaria symptoms, only $25 \%(n=10)$ would confirm the negative result with a BFM test. Most (70\%) would advise or perform a clinical diagnosis, $60 \%$ would not prescribe antimalarial medication to the patient, $50 \%$ would give another medicine, $28 \%$ would use additional diagnostic tools, and $18 \%$ would prescribe antimalarials anyway.

Table 3 Reasons stated for and against confirmation of a primary diagnosis 


\begin{tabular}{|c|c|c|c|c|c|c|c|c|}
\hline & \multicolumn{2}{|c|}{$\begin{array}{l}\text { Positive mRDT, } \\
\text { confirm with BFM? }\end{array}$} & \multicolumn{2}{|c|}{$\begin{array}{l}\text { Negative mRDT, } \\
\text { confirm with BFM? }\end{array}$} & \multicolumn{2}{|c|}{$\begin{array}{l}\text { Positive BFM, } \\
\text { confirm with } \\
\text { mRDT? }\end{array}$} & \multicolumn{2}{|c|}{$\begin{array}{l}\text { Negative BFM, } \\
\text { confirm with mRDT? }\end{array}$} \\
\hline & Obs. & $\%$ & Obs. & $\%$ & Obs. & $\%$ & Obs. & $\%$ \\
\hline No & 21 & 52.5 & 13 & 32.5 & 26 & 65.0 & 27 & 67.5 \\
\hline Yes & 19 & 47.5 & 27 & 67.5 & 14 & 35.0 & 13 & 32.5 \\
\hline Total & 40 & 100.0 & 40 & 100.0 & 40 & 100.0 & 40 & 100.0 \\
\hline \multicolumn{9}{|l|}{ Yes: reasons } \\
\hline Trust in BFM & 18 & 94.7 & 24 & 92.3 & & & & \\
\hline Trust in mRDT & & & & & 10 & 71.4 & 8 & 61.5 \\
\hline Other & 15 & 78.9 & 16 & 61.5 & 5 & 35.7 & 6 & 46.2 \\
\hline Trust in both tests & & & & & & & 6 & 46.2 \\
\hline No trust in result & 7 & 36.8 & 11 & 42.3 & 4 & 28.6 & 4 & 30.8 \\
\hline No trust in mRDT & 4 & 21.1 & 5 & 19.2 & & & & \\
\hline No trust in BFM & & & & & 4 & 28.6 & & \\
\hline $\begin{array}{l}\text { BFM difficult to } \\
\text { use }\end{array}$ & & & & & 7 & 50.0 & 3 & 23.1 \\
\hline Total respondents & 19 & 100.0 & 26 & 100.0 & 13 & 100.0 & 13 & 100.0 \\
\hline Total responses & 44 & & 56 & & 27 & & 27 & \\
\hline \multicolumn{9}{|l|}{ No: reasons } \\
\hline Trust in result & 21 & 100.0 & 13 & 100.0 & 24 & 92.3 & 26 & 96.3 \\
\hline Trust in mRDT & 18 & 85.7 & 12 & 92.3 & & & & \\
\hline Trust in BFM & & & & & 19 & 73.1 & 25 & 92.6 \\
\hline No trust in mRDT & & & & & 8 & 30.8 & 12 & 44.4 \\
\hline Trust in both tests & 4 & 19.0 & 6 & 46.2 & 4 & 15.4 & 3 & 11.1 \\
\hline $\begin{array}{l}\text { BFM difficult to } \\
\text { use }\end{array}$ & 2 & 9.5 & 4 & 30.8 & & & & \\
\hline $\begin{array}{l}\text { If symptomatic } \\
\text { you give medicine }\end{array}$ & 2 & 9.5 & 2 & 15.4 & & & 2 & 7.4 \\
\hline Other & 2 & 9.5 & 1 & 7.7 & 8 & 30.8 & 6 & 22.2 \\
\hline Total respondents & 21 & 100.0 & 13 & 100.0 & 26 & 100.0 & 27 & 100.0 \\
\hline Total responses & 49 & & 38 & & 63 & & 74 & \\
\hline
\end{tabular}


BFM, blood film microscopy; mRDT, malaria Rapid Diagnostic Test

When HCPs were asked if they would advise confirmation of a positive microscopy result with mRDT, the majority (65\%) answered no (Table 3). The top reasons given for advising against confirmation with mRDT were "trust in result" (92\%) and "trust in blood slide" (73\%), while the main reason given for advising for confirmation with mRDT was "trust in mRDT" (71\%). When asked if they would advise confirmation of a negative blood film result with a $\mathrm{mRDT}$, the majority of the respondents $(68 \%)$ answered no. The results were significantly associated with having received training for the use of $\operatorname{mRDT}(n=39 ; p=0.01)$. Confirmation of a negative blood film result with mRDT would not be advised by $77 \%$ of those having received mRDT training versus $25 \%$ of those not having received mRDT training (OR: 0.09 [95\% Cl: 0.009-0.747], $\mathrm{p}=0.01)$.

Inquiring about how HCPs perceived healthcare seekers'preferences of malaria diagnostics, more than half of the staff believed that healthcare seekers did not have a preference in any of the malaria diagnostic tests $(n=25 ; 63 \%)$. Of the remaining $33 \%(n=15)$ believing that healthcare seekers do have a preference, $80 \%$ felt that patients prefer microscopy testing, whereas only $20 \%$ felt that patients preferred mRDT. The reasons given for justifying the answers were that patients had "trust in blood slide" ( $n=12 ; 100 \%)$, patients had "no trust in mRDT" ( $n=8 ; 67 \%)$, patients would think that the "mRDT result is always negative" $(n=7 ; 58 \%)$ and that patients have "no knowledge on $\operatorname{mRDT}^{\prime \prime}(\mathrm{n}=3 ; 25 \%)$.

\section{Survey with household members}

\section{Characteristics of the households and household members}

In total, 309 household members were interviewed, mostly from households located in a village $82 \%$. A dispensary was the closest health facility for the majority of households (84\%) and in $78 \%$ of cases, the closest health facility was government owned. More than half of the households (52\%) were composed of 1-3 individuals, and the average household monthly income was 128,240 Tanzanian Shilling. Most respondents considered themselves either as the head of the household (47\%) or as the husband/wife of the household head (42\%). The mean age of the respondents was 40 years and the majority were females (68\%). Most respondents were married or living together $(76 \%)$, and $90 \%$ of respondents attended school with the highest level of education attained by $81 \%$ of respondents being primary education or post-primary training. The most-used source of information was the radio with $66 \%$ of respondents listening to it almost every day or at least once a week. One third (31\%) read newspapers/magazines and $22 \%$ watched television, at the same frequency. A total of $89 \%$ of respondents contributed to their household cash income. The most frequently stated means of contribution were agriculture and livestock (73\%) and petty business (37\%). Please consult Table S1 for full details of the household characteristics.

\section{Malaria perception among the respondents and their knowledge, attitudes and practices towards malaria} diagnostics

Malaria was considered by $66 \%$ of respondents to be a major health concern where they lived, $33 \%$ considered it a minor health concern and $1 \%$ did not know. Malaria perception was significantly associated with having ever attended school: malaria was perceived as a major health concern by $70 \%$ of the respondents that attended school and by $42 \%$ of those who did not (OR: 3.15 [95\% Cl: 1.38-7.31], $p=0.002$ ). Malaria perception was also significantly associated with the place of living: malaria was perceived as a major health concern in their area of living by $86 \%$ of those living in a district town, by $58 \%$ of those living in ward headquarters and by $65 \%$ of those living in a village (OR (district town vs. others): 3.42 [95\% Cl: 1.13-13.9], $\mathrm{p}=0.019)$. 
Among the interviewees, $63 \%$ knew that BFM was a method to test for malaria, while $28 \%$ were familiar with the mRDT and only $10 \%$ were aware of both testing methods. In total, $86 \%$ of interviewees said they would be interested in knowing more about the mRDT test. The majority of respondents (96\%) learned about malaria tests mainly from health facilities, $3 \%$ learned about the tests from the media and $0.3 \%$ through conversations with people.

Most respondents (68\%) were last tested for malaria between two and seven months from the date of the interview. Government-owned health facilities appeared to be the most common places for the respondents to be last tested ( $\mathrm{n}$ $=210,68 \%$ ) and the most common health facilities visited were dispensaries (39\%) (Table 4). In accordance, when asked to which facility respondents would choose to visit if they had malaria symptoms, government health facilities would be chosen by $79 \%$ and $51 \%$ of respondents would go to a dispensary.

Table 4 Choice of health facility

\begin{tabular}{|lll|}
\hline & Observation & $\%$ \\
\hline Health facility level tested for malaria the last time & & \\
\hline Dispensary & 120 & 38.8 \\
\hline District hospital & 107 & 34.6 \\
\hline Health center & 46 & 14.9 \\
\hline Private laboratory & 36 & 11.7 \\
\hline Total & 309 & 100.0 \\
\hline Health facility type tested for malaria the last time & & \\
\hline Government & 210 & 68.0 \\
\hline Private & 69 & 22.3 \\
\hline Faith-based & 30 & 9.7 \\
\hline Total & 309 & 100.0 \\
\hline Preferred health facility level when malaria symptoms present & & \\
\hline Dispensary & 156 & 51.3 \\
\hline District hospital & 89 & 29.3 \\
\hline Health center & 43 & 14.1 \\
\hline Private laboratory & 16 & 5.3 \\
\hline Total & 304 & 100.0 \\
\hline Preferred health facility type when malaria symptoms present & & \\
\hline Government & 240 & 78.9 \\
\hline Private & 37 & 12.2 \\
\hline Faith-based & 27 & 8.9 \\
\hline Total & 304 & 100.0 \\
\hline
\end{tabular}


The most frequently cited reason for choosing the last health facility that a respondent attended was the availability of diagnostic services ( $n=218,70.6 \%)$ followed by the perception of "good services" $(n=184,60 \%)$, that the facility was close by $(n=108,35 \%)$ and the service was affordable $(n=64,21 \%)$. Yet, in $73 \%$ of cases, the respondents were last tested for malaria in a health facility that was not the one closest to their household. Of the respondents that had a dispensary as the closest health facility to their household, $44 \%$ were tested for malaria there, whereas $35 \%$ preferred to go to the district hospital, $11 \%$ to a health centre and $11 \%$ to a private laboratory. Before going to the health facility where they were tested for malaria the last time, $14 \%$ of interviewees had visited another health facility which was in most cases a dispensary (81\%) and government-owned (78\%).

Prior to seeking treatment in a health facility, $75 \%$ of respondents did not take any medication. Among the $25 \%$ that did, 52\% took Paracetamol, 16\% Artemether and Lumefantrine (ALu)/Coartem, 13\% Sulfadoxine-pyrimethamine (SP). Only $2 \%$ of the respondents had purchased an mRDT for themselves before going to any health facility.

The most frequently used malaria test during the respondents' last visit to a health facility was BFM (66\%), whereas $34 \%$ were tested with an mRDT and only one respondent was tested with both tests. The test results $(n=306)$ were positive for $71 \%$ of respondents; $73 \%$ of those tested using the BFM tested positive for malaria and $67 \%$ of those tested using a mRDT tested positive for malaria. More than three quarters of respondents said they were highly satisfied with the treatment received after testing for malaria (76\%), 20\% were fairly satisfied and only $4 \%$ were not satisfied.

\section{Preference of malaria testing methods}

More than half of the respondents (58\%), if presented with malaria symptoms, stated that they would prefer to be tested by BFM, whereas $23 \%$ would rather be tested with an mRDT and $18 \%$ did not have a particular preference. Only two respondents stated that they would like to be tested with a combination of both tests and two respondents did not know.

Among those having a preference for microscopy, the four most frequently given reasons were "high diagnostic standard" (84\%), "trust in test/result" (61\%), the interviewee "knows only this one test" (28\%) and it is an "old test" (23\%). The most frequently cited reasons for preferring mRDT were that they provide a "quick result" (80\%), that it is a "quick service" (67\%) and mRDTs are perceived as a "new/modern test" (30\%).

\section{Trust in blood film microscopy}

There was a high level of trust in the accuracy of test results among those respondents that were last tested for malaria with BFM (96\%). The most cited reasons for trusting the test results were that they had received a "correct treatment plan" (71\%), that they had "trust in the test" (53\%), that they received a "positive test result" (50\%) and that it "confirmed assumptions" (33\%). The most cited reasons for not trusting the test result were that they had received a "negative result" (75\%), that "symptoms remain" (75\%) and it "did not confirm an assumption" (63\%). When asked whether they would get tested again with BFM, $86 \%$ of interviewees said that they would.

\section{Trust in $m R D T$}

The level of trust in the test result was very high (87\%) among those that were last tested for malaria with an mRDT. The most frequently stated reasons for trusting the test results were that they had received a "correct treatment plan" 
(63\%), a "positive test result" (43\%), that they "trust in the test" (40\%) and it "confirmed an assumption" (35\%). The most frequently given reasons for not trusting the mRDT result were that they had no trust in the results (79\%), having received "negative results" (43\%) and that the "symptoms remain" (43\%).

As was the case for malaria diagnosis with BFM testing, a proportion of those that declared to have trust in the mRDT result said they would not get tested with mRDT again. Paradoxically, the most frequently mentioned reason for not wanting to get tested again with mRDT was the delayed result (64\%).

\section{Behaviour following test results}

Among the respondents who, at the beginning of the interview, said they were only aware of mRDT as a malaria diagnostic tool, $82 \%$ would seek a second opinion if the test result was negative. In contrast, if the test result was positive only $9 \%$ of the respondents would seek a second opinion. Similarly, the vast majority (83\%) of those who were only aware of BFM diagnostics would seek a second opinion in the case of a negative blood film result, whereas only $9 \%$ would do so in the case of a positive blood film result. Among those familiar with both malaria tests, $90 \%$ would seek a second opinion with a negative mRDT and $20 \%$ would do so in the case of a positive mRDT result. For BFM testing, 45\% would seek a second opinion if faced with a negative blood film result and $10 \%$ of respondents would seek a second opinion in the case of a positive diagnostic result.

\section{Discussion}

The perception of malaria as a major health concern differed remarkably between the interviewed HCPs and healthcare seekers in the district of Kondoa. Whereas the majority of household interviewees perceived malaria as a major health concern, in accordance with the Malaria Indicator Survey from 2012 [43], less than one third of HCPs had the same opinion. The latter perceptions could be considered a reflection of the reduction in malaria incidence in Tanzania from 343.7 per 1000 population at risk in 2000 to 122.1 per 1000 population at risk in 2012 [44].

According to the health facilities' logbooks, more than $20 \%$ of all malaria diagnoses relied solely on a clinical diagnosis between 2013 and 2014 thus increasing the likelihood in this group of being over-diagnosed with malaria and incorrectly treated with antimalarials when suffering with non-malaria febrile illnesses that express similar symptoms to malaria [15]. Furthermore, there were a higher number of patients tested by BFM $(n=7795,45 \%)$ with a much higher percentage of patients testing positive for malaria $(71 \%)$ compared to those tested with mRDT ( $\mathrm{n}=$ $5709,33 \%)$ with a much lower rate of malaria positivity (14\%). Considering the challenges of microscopy as a diagnostic tool for malaria in rural Tanzania, the substantial number of patients that tested positive with BFM would have exacerbated the number of incorrect malaria diagnoses and treatments prescribed together with the potentially false positive clinical diagnoses $[17,18]$.

Comparing the diagnostic tools used on the last visit to a health facility for a malaria test, according to the responses of household members that were interviewed, BFM was used in $66 \%$ of cases with $72 \%$ of those testing positive, a similar proportion of positive tests to the data collected from health facility logbooks between 2013 and 2014. The proportion of patients tested with BFM was very high considering that the majority of respondents (68\%) were tested for malaria in a government-owned health facility, where the Ministry of Health guidelines indicated that patients should be tested for malaria with mRDTs. Household interviewees reported receiving an mRDT in only $34 \%$ of cases on the last visit to a health facility for a malaria test, and $67 \%$ of those reported having a positive test result. Albeit the overall percentage of mRDTs reported here were similar to the retrospective health facility data collected, the percentage of positive mRDT results, according to interviewees on their last health facility visit, was much higher

Page $15 / 22$ 
than the $14 \%$ positivity result retrieved from the health facilities records. The only explanation we have for this is a potential bias at the moment of data collection and that a number of negative mRDT test results may have been given a clinical malaria diagnosis and the respondents then stated a positive mRDT result.

The average availability of mRDTs over the 12-month period in all facilities was sub-optimal (63\%). Focusing on the government-owned health facilities only, they produced a slightly better average of $64 \%$ over the 12 -month period but a concerning $44 \%$ average availability during the rainy season when malaria incidence increases, as does the demand for mRDTs.

The decreasing availability of mRDTs in health facilities over the 12-month period, due to delayed deliveries and stock-outs among others, is likely to have contributed to HCPs using clinical diagnosis and BFM as a means of testing for malaria to cope with demands [36].

Where more than half of the HCPs thought that patients had no malaria diagnostic preference, the majority of patients (81\%) expressed a clear preference for BFM. Less than a third had any knowledge of mRDT as a malaria diagnostic tool. Although the level of trust in BFM was extremely high (96\%) among those that were last diagnosed with BFM and the level of trust in mRDT was high among those last diagnosed with mRDTs, $75 \%$ of BFM interviewees and $79 \%$ of $\mathrm{mRDT}$ interviewees would not trust their respective results if they presented as negative. Equally, the results showed that for those who had knowledge of both tests, the level of trust was highly influenced by the test result. A negative result was more likely to be accepted if it was diagnosed with a microscope compared to a negative result from an $\mathrm{mRDT}$ and in the latter case there would be a stronger wish for confirmation of a negative test result.

Less than half of healthcare providers preferred using BFM as a trusted testing method, $57 \%$ of those considered it more accurate than an mRDT and $42 \%$ did not trust mRDTs. Conversely, less than half of HCPs preferred the use of mRDTs mainly due to their simplicity and speed, whereas $77 \%$ of those considered they were more accurate than BFM. Although the majority of HCPs trusted the mRDT test result, 70\% would provide a clinical diagnosis in the case of a negative mRDT with symptoms typical for malaria. This behaviour could be a reflection of conformity to much older and more confusing malaria diagnosis and treatment guidelines from 2006 that suggested using clinical judgement when a test result was negative $[36,45,46]$. However, given the many challenges HCPs face adhering to treatment guidelines in Tanzania $[11,19,47]$, a complexity of other more tangible reasons could be considered more compelling, such as a need to provide a diagnosis and treatment and patient pressure to provide one [15, 19]. In cases where HCPs could confirm mRDT results with a blood film, the majority would do so, particularly if the mRDT result was negative (68\%). In contrast, where HCPs could confirm BFM test results with an mRDT, approximately twothirds would not, whether the blood slide presented a positive or negative result. Although the question was hypothetical (i.e. not observed and documented in this study), the HCPs responses led to the assumption that they have higher trust in blood film results compared to mRDT results and generally have a higher level of trust if results are positive. Of course, this difference in opinion between HCPs' confidence of one testing method over another, despite treatment guidelines advocating different diagnosis and treatment, could be confusing to patients and potentially have a profound influence over their choice and trust in a particular method of testing. Non-conformity to malaria treatment guidelines and compliance to negative mRDT results by HCPs in Tanzania will probably continue to be a problem until the capacity of HCPs to diagnose other febrile illnesses peculiar to a locality, paired with increased options for laboratory tests to rule out other reasons, has improved $[10,11,15]$. Continued and more focused training and supportive supervision of HCPs, especially when new treatment guidelines are introduced, should include the diagnosis and treatment of non-malaria febrile illnesses that include area specific illnesses. This

Page $16 / 22$ 
can help provide HCPs with options to treat a patient that test negative for malaria and prescribe more suitable drugs to prevent the over use of malaria medication to help combat resistance to malaria medication and over use of limited resources. Positive uniform messages, reinforced from frequently trained HCPs, in addition to public behaviour change approaches could provide patients with a sense of security in accepting and adhering to WHO guidelines on malaria approved by the Tanzanian Ministry of Health [33].

\section{Limitations}

The presented study was conducted in Kondoa district, which differs in malaria prevalence, ethnic composition, geography and income sources compared to other districts in Tanzania. Due to these differences and a relatively small sample size among healthcare workers $(n=40)$, results cannot be attributed to the overall population of Tanzania.

Household members were included in the study if tested for malaria within the last 12 months, causing the potential for recall bias. To decrease the possibility of bias, interviewees were probed by showing them a blood film and mRDT device in case of uncertainty at the beginning of the interview. To reduce recall bias for selected questions providing multiple reply options, interviewees were first asked to answer spontaneously and following, reply options were read out. To reduce interviewer bias, on the other hand, data collectors received intensive training prior to the study commencement, which included a pilot study.

Questions relating to the confirmation of test results were hypothetical and not validated by observations and replies and therefore need to be interpreted with caution.

Paper-based questionnaires have the potential to decrease data quality due to data entry mistakes. To ensure data entry of the highest quality, data was entered twice by a team of two.

Finally, the study was concluded in 2014 , therefore, the article presents data that may not accurately reflect the current situation. Nevertheless, subsequent observations and the authors' continued experience in Tanzania indicates that the situation has not significantly changed. Moreover, the current study fills an important gap in the scientific literature owing to the scarcity of relevant studies in this area across Tanzania and may serve as a comprehensive orientation to future interventions in the clinical management of malaria - most notably in the area of diagnostics and adherence to standard treatment guidelines. To the best of our knowledge, this is still the only study that comprehensively covers the knowledge, attitudes and practices towards malaria diagnostics among both healthcare providers and healthcare seekers in Tanzania.

\section{Conclusions}

Frequent malaria and non-malaria febrile illness diagnosis and treatment training for HCPs is essential for healthcare providers to offer uniform, quality services that patients can trust. This requires regular mRDT training for rural HCPs in addition to reinforcing good microscopy practice where they are used in quality assured conditions. HCPs adherence to the latest guidelines for treating patients presenting with malaria symptoms needs to be encouraged whether or not a negative test result ensues. As private health facilities and laboratories prove popular for malaria testing, all governmental and private health facilities require a uniform approach that could be directed by guidelines and/or by law. Malaria diagnosis and treatment guidelines, therefore, may need to be simplified and made available in Swahili for the lower cadres of HCPs to comprehend and advocate to the public. The onus on the public to adhere to correct diagnoses and treatments can also be reiterated and reinforced by tailored

Page $17 / 22$ 
communication and behaviour change interventions with various forms of media and outreach that can target groups that are more in need, namely the poor and illiterate. Provided mRDT distribution is strengthened to avoid stock outs, especially in the malaria peak season, these measures could have the potential of reducing the high number of clinically diagnosed malaria cases and inadequate quality microscopy diagnoses, thereby reducing antimalarial prescriptions where they are not necessary and supporting the fight against antimalarial resistance. These findings emphasize the need for a sensitive approach when introducing new diagnostic tools. It shows the need for the continuous provision of delivering knowledge and training for HCPs to establish the needed trust in test results and sharing of information for healthcare seekers at the community level.

\section{Abbreviations}

mRDT: Malaria rapid diagnostic test; HCP: healthcare provider; WHO: The World Health Organization; Tanzania: The United Republic of Tanzania; ACT: artemisinin-based combination therapy; HPSS: The Health Promotion and System Strengthening Project; OR: odds ratios; Cl: confidence interval; Swiss TPH: Swiss Tropical and Public Health Institute; SDC: Swiss Agency for Development and Cooperation; BMF: blood film microscopy; NIMR: National Institute of Medical Research; EKNZ: Ethikkommission Nordwest- und Zentralschweiz; ICF: Information and Consent Form

\section{Declarations}

\section{Ethics approval and consent to participate}

Ethical clearance was obtained from the National Institute for Medical Research (NIMR) (NIMR/HQ/R.8a/Vol. IX/1689) in Tanzania for the full study and from the Ethikkommission Nordwest- und Zentralschweiz (EKNZ) (EKNZ: 2014-021) in Switzerland for aspects of the study pertaining to research quality. The Regional Medical Officer and District Medical Officer provided approval prior to data collection.

\section{Consent for publication}

All participants included in this study received an Information and Consent Form (ICF) in Swahili prior to data collection and provided informed written or fingerprint consent. In case of illiteracy, the ICF was read out by an impartial witness.

\section{Availability of data and material}

The datasets used and/or analysed during the current study are available from the corresponding author on reasonable request.

\section{Competing interests}

The authors declare that they have no competing interests.

\section{Funding}

The study was conducted on behalf of the Health Promotion and System Strengthening Project (HPSS) in Dodoma Region, Tanzania. Swiss TPH was mandated with the implementation of this project, which is funded by the Swiss Agency for Development and Cooperation (SDC) (Grant number Phase 7F-07381.01.02, Contract No. 81013135). The funding body did not have any influence on the design of the study including data collection, analysis, and interpretation of data and in writing the manuscript. 


\section{Authors' contributions}

LFB conceptualized the study with input from KMO and AKA. AKA and LFB were responsible for data collection in the field supported by a research team. FGA analysed the data and developed the graphics with support of LFB. LFB wrote the article with contributions by AKA, FGA, KMO and RC. All authors read and approved the final draft of the manuscript.

\section{Acknowledgements}

We would like to thank the ethics committee of the Ethikkommission Nordwest und Zentralschweiz (EKNZ) and the National Institute for Medical Research (NIMR) for their review and granting of ethical clearance. Further we would like to thank the Study Secretariat of the Swiss Tropical and Public Health Institute (Swiss TPH) for advice on the ethical clearance proposal, namely Monique Vogel. We would like to thank the Swiss Agency for Development and Cooperation (SDC) for funding of this study within the frame of the HPSS project. The study could not have been realized without the support of Vicky-Sidney Msamba, the Monitoring, Evaluation and Operational Research Officer, Dr Ezekile Mpuya, the Regional Medical Officer of Dodoma region, Dr James Charles and Dr John Luanda, the District Medical Officer in charge. We would also like to thank the entire team of HPSS and the five research assistants, Catherine Mungure, Emmanuel John, Happy Ngingo and Griffin Richard. We thank all healthcare providers and healthcare seekers, who participated in the study and shared their time and valuable insights; without them, the study could not have been realized. Finally, we thank Dr Jasmina Saric for support during the write-up and publishing process.

\section{References}

1. Millenium Development Goals (MDG) and beyond 2015 [https://www.un.org/millenniumgoals/]

2. World Health Organization. World Malaria Report 2020. In. Geneva; 2020.

3. The Roll Back Malaria Partnership. The global malaria action plan for a malaria free world. In. Geneva: United Nations Office for Project Services; 2008.

4. World Health Organization. Global technical strategy for malaria 2016-2030. In. Geneva: World Health Organization; 2015.

5. Snow RW, Sartorius B, Kyalo D, Maina J, Amratia P, Mundia CW, Bejon P, Noor AM. The prevalence of Plasmodium falciparum in sub-Saharan Africa since 1900. Nature. 2017;550(7677):515-+.

6. Tanzania population [https://www.worldometers.info/world-population/tanzania-population/]

7. USAID. President's malaria initiative Tanzania: Malaria Operational Plan FY 2019 In.; 2019.

8. World Health Organization. Guidelines for the treatment of malaria: 3rd edition. In. Geneva; 2006.

9. mal ERARCPoHS, Policy R. malERA: An updated research agenda for health systems and policy research in malaria elimination and eradication. PLoS Med. 2017;14(11):e1002454.

10. Kabaghe AN, Visser BJ, Spijker R, Phiri KS, Grobusch MP, van Vugt M. Health workers' compliance to rapid diagnostic tests (RDTs) to guide malaria treatment: a systematic review and meta-analysis. Malaria J. 2016;15.

11. Budimu A, Emidi B, Mkumbaye S, Kajeguka DC. Adherence, Awareness, Access, and Use of Standard Diagnosis and Treatment Guideline for Malaria Case Management among Healthcare Workers in Meatu, Tanzania. J Trop Med-Us. 2020;2020.

12. USAID. President's Malaria Initiative Tanzania: Malaria Operational Plan FY 2013. In.; 2013. 
13. Mbonye AK, Magnussen P, Lal S, Hansen KS, Cundill B, Chandler C, Clarke SE. A Cluster Randomised Trial Introducing Rapid Diagnostic Tests into Registered Drug Shops in Uganda: Impact on Appropriate Treatment of Malaria. Plos One. 2015;10(7).

14. Steinhardt LC, Chinkhumba J, Wolkon A, Luka M, Luhanga M, Sande J et al. Patient-, health worker-, and health facility-level determinants of correct malaria case management at publicly funded health facilities in Malawi: results from a nationally representative health facility survey. Malaria J. 2014;13.

15. Chipwaza B, Mugasa JP, Mayumana I, Amuri M, Makungu C, Gwakisa PS. Community Knowledge and Attitudes and Health Workers' Practices regarding Non-malaria Febrile Illnesses in Eastern Tanzania. Plos Neglect Trop D. 2014;8(5).

16. USAID. U.S President's malaria initiative Tanzania(Mainland): Malaria Operational Plan FY2020. In.; 2020.

17. Harchut K, Standley C, Dobson A, Klaassen B, Rambaud-Althaus C, Althaus F, Nowak K. Over-diagnosis of malaria by microscopy in the Kilombero Valley, Southern Tanzania: an evaluation of the utility and costeffectiveness of rapid diagnostic tests. Malaria J. 2013;12.

18. World Health Organization. WHO guidelines for malaria. In. Geneva; 2021.

19. Kwesigabo G, Mwangu MA, Kakoko DC, Warriner I, Mkony CA, Killewo J, Macfarlane SB, Kaaya EE, Freeman P. Tanzania's health system and workforce crisis. J Public Health Pol. 2012;33:S35-S44.

20. Kahama-Maro J, D'Acremont V, Mtasiwa D, Genton B, Lengeler C. Low quality of routine microscopy for malaria at different levels of the health system in Dar es Salaam. Malaria J. 2011;10.

21. Abba K, Deeks JJ, Olliaro P, Naing CM, Jackson SM, Takwoingi Y, Donegan S, Garner P. Rapid diagnostic tests for diagnosing uncomplicated P. falciparum malaria in endemic countries. Cochrane Db Syst Rev. 2011(7).

22. Wongsrichanalai C, Barcus MJ, Muth S, Sutamihardja A, Wernsdorfer WH. A review of malaria diagnostic tools: Microscopy and rapid diagnostic test (RDT). Am J Trop Med Hyg. 2007;77(6):119-127.

23. Chandler CIR, Meta J, Ponzo C, Nasuwa F, Kessy J, Mbakilwa H, Haaland A, Reyburn H. The development of effective behaviour change interventions to support the use of malaria rapid diagnostic tests by Tanzanian clinicians. Implement Sci. 2014;9.

24. Ruizendaal E, Dierickx S, Grietens KP, Schallig HDFH, Pagnoni F, Mens PF. Success or failure of critical steps in community case management of malaria with rapid diagnostic tests: a systematic review. Malaria J. 2014;13.

25. Edson F, Kayombo EJ. Knowledge on malaria transmission and its prevention among schoolchildren in Kyela District, south-western Tanzania. Tanzan Health Res Bull. 2007;9(3):207-210.

26. Kamat VR. "I thought it was only ordinary fever"! cultural knowledge and the micropolitics of therapy seeking for childhood febrile illness in Tanzania. Soc Sci Med. 2006;62(12):2945-2959.

27. Kinung'hi SM, Mashauri F, Mwanga JR, Nnko SE, Kaatano GM, Malima R, Kishamawe C, Magesa S, Mboera LE. Knowledge, attitudes and practices about malaria among communities: comparing epidemic and non-epidemic prone communities of Muleba district, North-western Tanzania. BMC Public Health. 2010;10:395.

28. Malisa AL, Ndukai M. Knowledge and practices on malaria and its control among pastoralists in Simanjiro District, northern Tanzania. Tanzan J Health Res. 2009;11(4):219-225.

29. Massele AY, Sayi J, Nsimba SED, Oforiadjei D, Laing RO. Knowledge and Management of Malaria in Dar-EsSalaam, Tanzania. E Afr Med J. 1993;70(10):639-642.

30. Mazigo HD, Obasy E, Mauka W, Manyiri P, Zinga M, Kweka EJ, Mnyone LL, Heukelbach J. Knowledge, Attitudes, and Practices about Malaria and Its Control in Rural Northwest Tanzania. Malar Res Treat. 2010;2010:794261. 
31. Ministry of Health CD, Gender, Elderly and Children (MoHCDGEC) [Tanzania Mainland], Ministry of Health (MoH) [Zanzibar], National Bureau of Statistics (NBS), Office of the Chief Government Statistician (OCGS), and ICF,. Tanzania Demographic and Health Survey and Malaria Indicator Survey (TDHS-MIS) 2015-16. In. Dar es Salaam, Tanzania, and Rockville, Maryland, USA; 2016.

32. Bruxvoort K, Kalolella A, Nchimbi H, Festo C, Taylor M, Thomson R et al. Getting antimalarials on target: impact of national roll-out of malaria rapid diagnostic tests on health facility treatment in three regions of Tanzania. Trop Med Int Health. 2013;18(10):1269-1282.

33. Hutchinson E, Reyburn H, Hamlyn E, Long K, Meta J, Mbakilwa H, Chandler C. Bringing the state into the clinic? Incorporating the rapid diagnostic test for malaria into routine practice in Tanzanian primary healthcare facilities. Glob Public Health. 2017;12(9):1077-1091.

34. Masanja IM, Selemani M, Amuri B, Kajungu D, Khatib R, Kachur SP, Skarbinski J. Increased use of malaria rapid diagnostic tests improves targeting of anti-malarial treatment in rural Tanzania: implications for nationwide rollout of malaria rapid diagnostic tests. Malaria J. 2012;11.

35. Williams HA, Causer L, Metta E, Malila A, O'Reilly T, Abdulla S, Kachur SP, Bloland PB. Dispensary level pilot implementation of rapid diagnostic tests: an evaluation of RDT acceptance and usage by providers and patients - Tanzania, 2005. Malaria J. 2008;7.

36. Mubi M, Kakoko D, Ngasala B, Premji Z, Peterson S, Bjorkman A, Martensson A. Malaria diagnosis and treatment practices following introduction of rapid diagnostic tests in Kibaha District, Coast Region, Tanzania. Malaria J. 2013;12.

37. Welfare TURoTMoHaS. National Malaria Control Programme: Communication guide for malaria control interventions 2015-2020. In: Malaria control series 31. 2015.

38. Diggle E, Asgary R, Gore-Langton G, Nahashon E, Mungai J, Harrison R et al. Perceptions of malaria and acceptance of rapid diagnostic tests and related treatment practises among community members and health care providers in Greater Garissa, North Eastern Province, Kenya. Malaria J. 2014;13.

39. Health Promotion and System Strengthening project (HPSS)

[https://issuu.com/communications.swisstph/docs/hpss_brochure]

40. Wiedenmayer K, Mbwasi R, Mfuko W, Mpuya E, Charles J, Chilunda F, Mbepera D, Kapologwe N. Jazia prime vendor system- a public-private partnership to improve medicine availability in Tanzania: from pilot to scale. J Pharm Policy Pract. 2019;12(1).

41. Kapologwe NA, Kagaruki GB, Kalolo A, Ally M, Shao A, Meshack M et al. Barriers and facilitators to enrollment and re-enrollment into the community health funds/Tiba Kwa Kadi (CHF/TIKA) in Tanzania: a cross-sectional inquiry on the effects of socio-demographic factors and social marketing strategies. Bmc Health Serv Res. 2017;17.

42. Bostoen K, Chalabi Z. Optimization of household survey sampling without sample frames. Int J Epidemiol. 2006;35(3):751-755.

43. Tanzania Commission for AIDS (TACAIDS) ZACZ, National Bureau of Statistics (NBS), Office of the Chief Government Statistician (OCGS), and ICF International 2013,. Tanzania HIV/AIDS and Malaria Indicator Survey 2011-12 In. Dar es Salaam, Tanzania: TACAIDS, ZAC, NBS, OCGS, and ICF International; 2013.

44. The global health observatory, world health data platform, United Republic of Tanzania [https://www.who.int/data/gho/data/countries/country-details/GHO/united-republic-of-tanzania? countryProfileld=40f06adc-047c-435c-b576-ebd4da789bb2] 
45. National Malaria Control Programme. National guidelines for malaria diagnosis and treatment. In. Edited by Welfare MoHaS. United Republic of Tanzania; 2006.

46. D’Acremont V LC, Genton B,. Stop ambiguous messages on malaria diagnosis. BMJ 2007;334:489.

47. Wiedenmayer K, Ombaka E, Kabudi B, Canavan R, Rajkumar S, Chilunda F, Sungi S, Stoermer M. Adherence to standard treatment guidelines among prescribers in primary healthcare facilities in the Dodoma region of Tanzania. Bmc Health Serv Res. 2021;21(1).

\section{Figures}

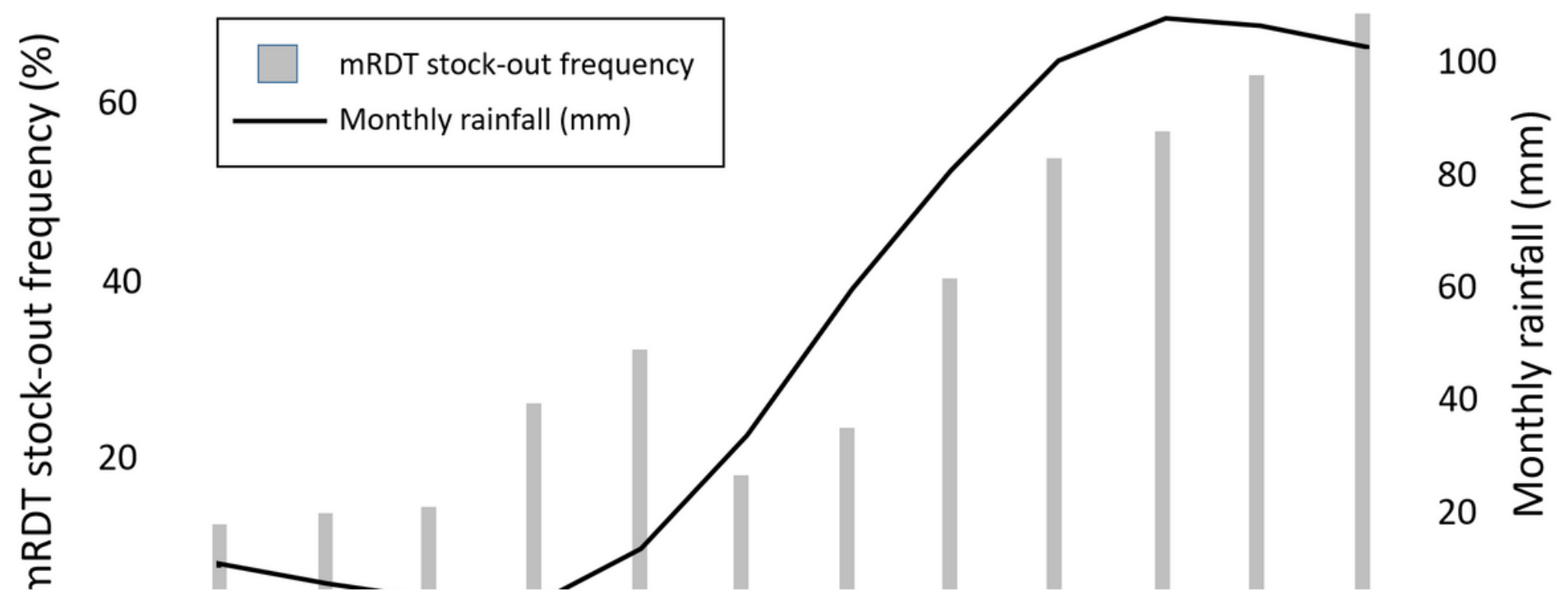

Figure 1

MRDT stock-outs and monthly rainfall in Kondoa over a 12-month inventory period 